\title{
Heterocyclization of polarized system:
} synthesis, antioxidant and anti-inflammatory 4-(pyridin-3-yl)-6-(thiophen-2-yl) pyrimidine-2-thiol derivatives

\author{
Wesam S. Shehab ${ }^{1^{*}} \mathbb{D}$, Magda H. Abdellattif ${ }^{2}$ and Samar M. Mouneir ${ }^{3}$
}

\begin{abstract}
Background: Chalcones are intent in the daily diet as a favorable chemotherapeutic compound; on the other hand thiophene moiety is present in a large number of bioactive molecules having diverse biological efficiency.

Results: Our current goal is the synthesis of (E)-1-(pyridin-3-yl)-3-(thiophen-2-yl) prop-2-en-1-one $\mathbf{3}$ that's used as a starting compound to synthesize the novel pyrimidine-2-thiol, pyrazole, pyran derivatives. Chalcones $\mathbf{3}$ was prepared by condensation of 3-acetylpyridine with thiophene 2-carboxaldehyde which reacted with thiourea to obtain pyrimidinthiol derivative $\mathbf{4}$. Compound $\mathbf{4}$ was allowed to react with hydrazine hydrate to afford 2-hydrazinylpyrimidine derivative $\mathbf{5}$. Compound $\mathbf{5}$ was used as a key intermediate for a facile synthesis of the targets $\mathbf{6}$ and $\mathbf{7}$. In contrast, pyranone $\mathbf{8}$ was obtained by transformation of compound $\mathbf{5}$. Using as a precursor for the synthesis of new pyrazolo pyrimidine derivatives $\mathbf{9} \mathbf{- 1 0}$. The major incentive behind the preparation of these compounds was the immense biological activities associated to these heterocyclic derivatives.
\end{abstract}

Conclusions: The newly synthesized compounds (1-4) showed potent anti-inflammatory activities both in vitro and in vivo. They also exhibited promising antioxidant vitalities against $a$, $a$-diphenyl- $\beta$-picrylhydrazyl scavenging activity and lipid peroxidation. In conclusion, compound $\mathbf{1}$ showed a hopefully anti-inflammatory and antioxidant activities.

Keywords: Pyrazolopyrimidine, Thiophene, Chalcone, Pyrazol, Pyranone, Anti-inflammatory-antioxidantcycloxygenase-5-LOX-DPPH

\section{Introduction}

Chalcones are distinguished by their easy synthesis from Claisen-Schmidt condensation. The chemical structure of chalcones formed of two aromatic rings joined by a thee carbon, $\alpha, \beta$-unsaturated carbonyl system (1,3-diphenylprop-2-en-1-one) [1, 2]. They have been authenticated with diverse biological efficiency including antibacterial [3-8], anti-inflammatory [9-12], antioxidant [13-16], anti-tumor effects [17-22]. Also, pyridine derivatives of different heterocyclic nucleus have shown potent

\footnotetext{
*Correspondence: wsshehab@zu.edu.eg; Wesamshehab2015@gmail. com

${ }^{1}$ Department of Chemistry, Faculty of Science, Zagazig University, Zagazig 44519, Egypt

Full list of author information is available at the end of the article
}

pharmacological properties like cytotoxic activity [23, 24]. Recent studies have demonstrated that chalcones are target in the daily diet as a favorable chemotherapeutic compounds [25] and anti-proliferative activity [26]. On the other hand thiophene moiety is present in a large number of bioactive molecules having diverse biological activities such as anti-inflammatory [27], anticonvulsant [26], antimicrobial [27] and antitumor [28]. Moreover, thiophene moiety is a well-known isostere for benzene; for example, the replacement of benzene ring of the antidepressant drug, Viloxazine led to a prolongation of the half-life [29]. Recently we were concerned with the synthesis of polyfunctional heterocyclic compounds, where the (E)-1-(pyridin-3-yl)-3-(thiophen-2-yl) prop-2-en-1one 3 was used as a starting compound. The remarkable 
biological activity of the polycyclic heterocyclic compounds encouraged us to continue our previous work on the synthesis of fused pyrimidine [30-33] and their applications, by designing a polycyclic heterocyclic compounds containing five and/or six rings fused with each other to develop a superior biological activity.

\section{Results and discussion \\ Chemistry}

Aldol condensation reaction of 3-thiophenecarboxaldehyde $\mathbf{1}$ with 3 -acetylpyridine $\mathbf{2}$ in ethanolic $\mathrm{NaOH}$ solution afforded chalcone 3 . The structure of compound 3 was elucidated by its IR, ${ }^{1} \mathrm{H}$ NMR and ${ }^{13} \mathrm{C}$ NMR. Its IR spectrum showed a characteristic peak for a conjugated carbonyl group at $1633 \mathrm{~cm}^{-1}$, and by its ${ }^{1} \mathrm{H}$ NMR which gave signals at $\delta 7.53(\mathrm{~d}, 1 \mathrm{H}, J=12.9 \mathrm{~Hz}$, $(\mathrm{CH}=\mathrm{C}-\mathrm{C}=\mathrm{O})$, and $7.92(\mathrm{~d}, 1 \mathrm{H}, J=12.9 \mathrm{~Hz}(\mathrm{CH}=\mathrm{C}-$ $\mathrm{C}=\mathrm{O}$ ) and two doublet signals at $\delta=7.28$ and 7.94 due to thiophenyl- $\mathrm{C}_{4} H$ and thiophenyl- $\mathrm{C}_{3} H$ and another at 8.11 owing to thiophenyl- $C_{5}, H$ whereas, the ${ }^{13} \mathrm{C}$ NMR spectrum showed a signal at ( $\delta$ in ppm) 123 caused by ethylene group and 125, 126, 135, 149 and a signal due to $\mathrm{C}=\mathrm{O}$ groups at 193 . [3+3] base induced cycloaddition of chalcone 3 with thiourea gave 4-(pyridin-3-yl)6-(thiophen-2-yl) pyrimidine-2 $(1 \mathrm{H})$-thione 4 . IR spectra of compounds 4 showed the presence of a $\mathrm{C}=\mathrm{S}$ band at $1270 \mathrm{~cm}^{-1}$ and an absorption band in the range 3433$3490 \mathrm{~cm}^{-1}$ attributed to the amine (NH). The ${ }^{1} \mathrm{H}$ NMR spectrum of compound 4 two doublet signals at $\delta=7.28$ and 7.94 due to thiophenyl- $\mathrm{C}_{4}, H$ and thiophenyl- $\mathrm{C}_{3}, H$ and another at 8.11 as a result of thiophenyl- $C_{5} H$. The spectra displayed a singlet at 8.82 for $\mathrm{NH}$, respectively.
The hydrazinopyrimidine derivative $\mathbf{5}$ was synthesized by condensation of the thiopyrimidinone $\mathbf{4}$ with hydrazine hydrate in refluxing alcohol, the structure of compound 5 was confirmed by the IR, ${ }^{1} \mathrm{H}$ NMR and elemental analysis, where its IR revealed the absorption bands at $v \max =3212$ for the $\mathrm{NH}_{2}$ and $3184 \mathrm{~cm}^{-1}$ for the $\mathrm{NH}$ group, ${ }^{1} \mathrm{H}$ NMR spectrum gave the signals at $\delta=8.93$ 8.95 as a broad singlet for $\mathrm{NH}_{2}$, hydrazine $\mathrm{NH}$, respectively (Scheme 1).

Cyclocondensation of chalcone 3 and ethyl cyanoacetate in the presence of sodium ethoxide under the reflux conditions [33] gave pyranone derivative 6. Condensation with hydrazinehydrate $[34,35]$ in refluxing ethanol leads to ring transformation producing corresponding pyridinones 7 . The structure of the target 7 was confirmed from its spectral data, where is IR spectra showed absorption bands in the region 2222 and $1688 \mathrm{~cm}^{-1}$ characteristic for $\mathrm{C} \equiv \mathrm{N}$ and carbonyl group, respectively (Scheme 2).

The hydrazinopyrimidine derivative $\mathbf{5}$ was used as a precursor for the synthesis of some heterocyclic compounds. The hydrazinopyrimidine derivative $\mathbf{5}$ reacted with ethyl acetoacetate in excess manner to afford compound $\mathbf{8}$. The formation of $\mathbf{8}$ may be proceeds via the formation of pyrazolone derivative $\mathbf{9}$ followed by the attack of methylene anion of pyrazolone to ketonic function of ethyl acetoacetate followed by pyran cyclization. IR spectrum of compound $\mathbf{8}$ revealed the absorption peaks at $1715 \mathrm{~cm}^{-1}$ characteristic of $\mathrm{C}=\mathrm{O}$ groups respectively, ${ }^{1} \mathrm{H}$ NMR exhibited the two singlets at $\delta=2.25$ and 2.32 for 2 $\mathrm{CH}_{3}$ protons and a singlet at $\delta=5.60 \mathrm{ppm}$ for pyranone $\mathrm{H}$. Furthermore, the pyrimidine pyrazolone compound

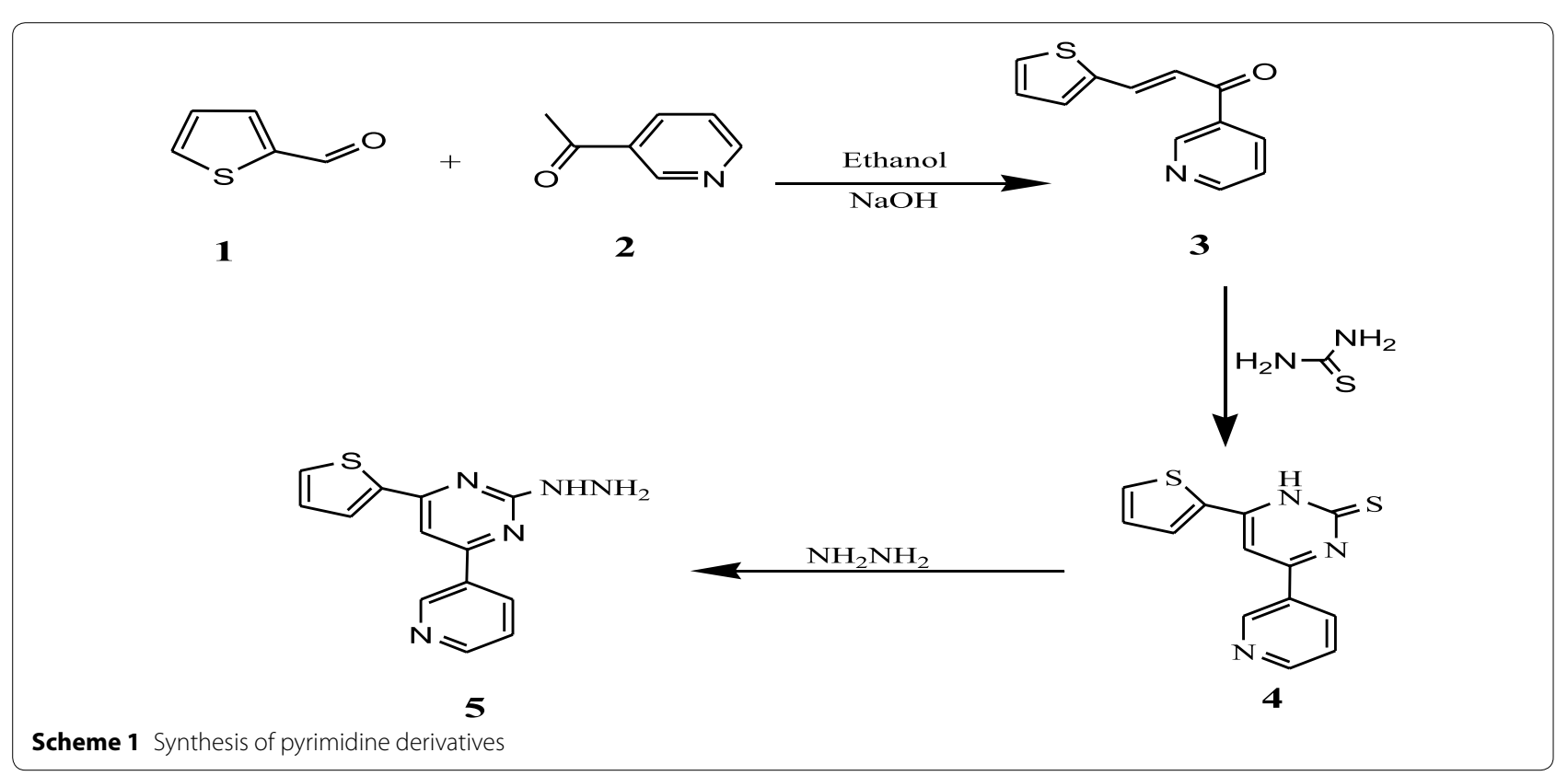




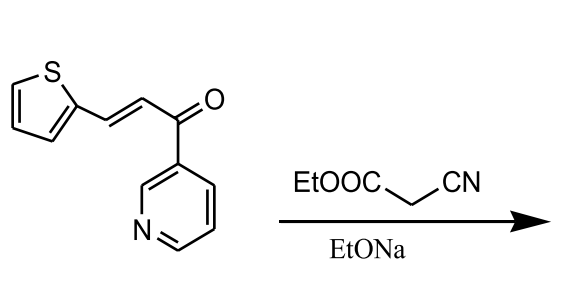

3

Scheme 2 Synthesis of pyranone and pyridinones derivatives<smiles>N#Cc1c(-c2cccs2)cc(-c2cccnc2)oc1=O</smiles>

6

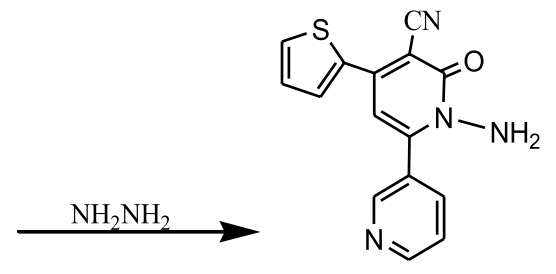

7

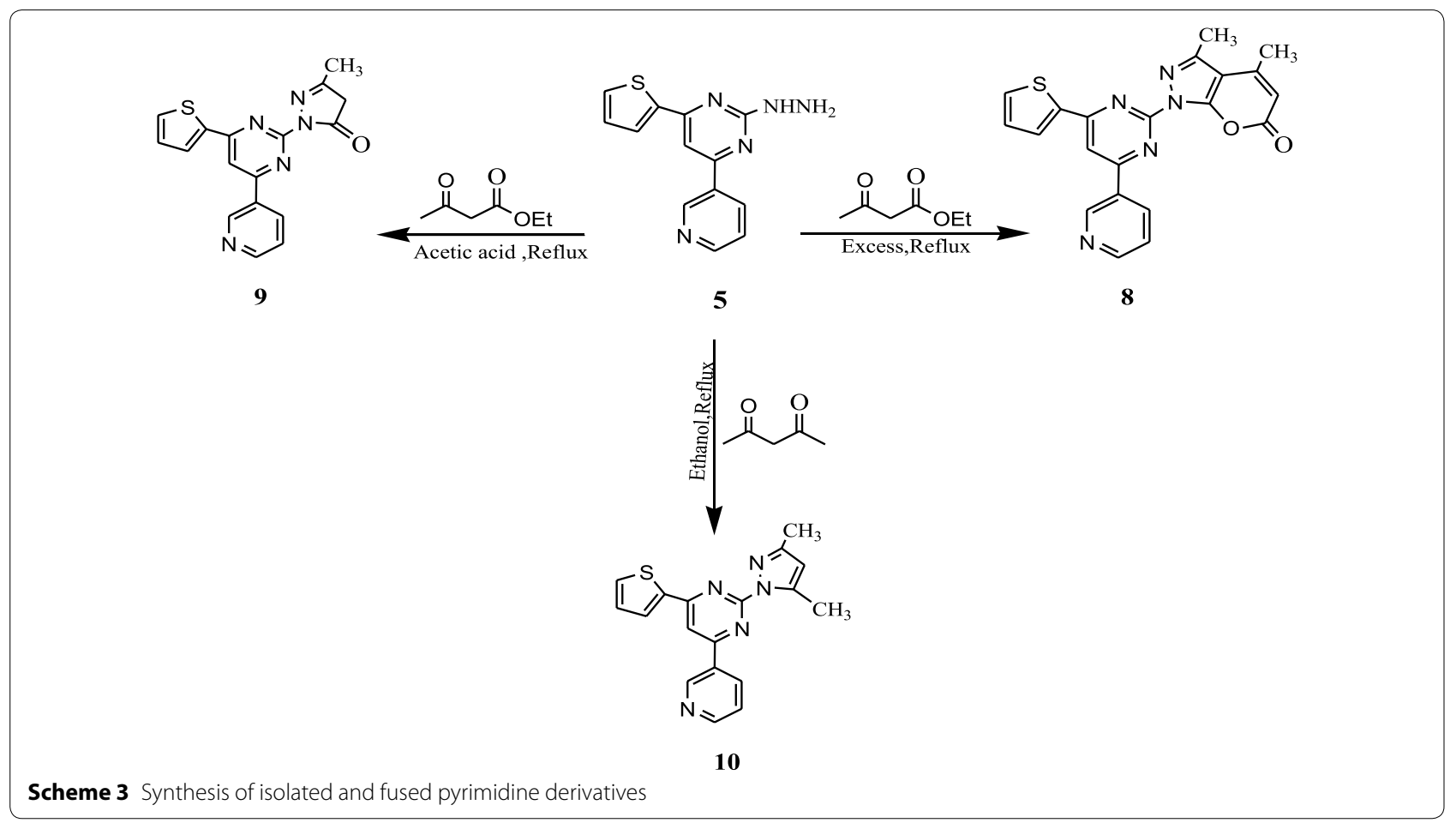

9 was obtained as a result of attack of hydrazinofunction of $\mathbf{5}$ to ethyl acetoacetate. The pyrazolo pyrimidine $\mathbf{1 0}$ was synthesized by heating an alcoholic solution of compound $5(10.0 \mathrm{mmol}$.) with acetylacetone $(10.0 \mathrm{mmol}$.) at reflux temperature for $5 \mathrm{~h}$. The IR spectra of $\mathbf{9}$ and $\mathbf{1 0}$ showed the disappearance of the hydrazine group where the ${ }^{1} \mathrm{H}$ NMR spectrum showed singlet pyrimidine $\mathrm{H}$ at $\delta=8.95 \mathrm{ppm}$ and two singlets for the two $\mathrm{CH}_{3}$ protons, respectively (Scheme 3 ).

\section{Biological activity studies}

\section{In vitro anti-inflammatory activity}

In vitro COX-1 and COX-2 inhibition Compounds (3-6) were calorimetrically evaluated for their anti-inflammatory activities in vitro for COX-1 and COX-2 at $590 \mathrm{~nm}$ using ovine COX-1/COX-2 inhibitor screening assay kit [36]. Celecoxib was used as a standard reference drug.

In vitro 5-LOX inhibition A bnova 5 lipoxygenase inhibitor screening assay was used [37]. Meclofenamate sodium was used as a standard reference drug. Results were expressed in Table 1 as IC ${ }_{50}$ as means of thee determinations the selectivity index was calculated also as $\mathrm{IC}_{50}$ $(\mathrm{COX}-1) / \mathrm{IC}_{50}(\mathrm{COX}-2)$.

\section{In vivo anti- inflammatory activity}

Carrageenan induced rat paw edema in rats: Fifty rats were divided into ten groups (i.e., each group, five rats). The first group (control), received carboxymethyl cellulose. The second group was given diclofenac sodium as a standard anti-inflammatory drug. Groups (3-10) were 
Table 1 Display the anti-inflammatory activity of the newly synthesized compounds as IC ${ }_{50}, \mu \mathrm{M}$ for COX1, COX-2 and 5-LOX

\begin{tabular}{|c|c|c|c|c|}
\hline Group & $\begin{array}{l}\mathrm{IC}_{50}(\mu \mathrm{M}) \\
\text { COX-1 }\end{array}$ & $\begin{array}{l}\mathrm{IC}_{50}(\mu \mathrm{M}) \\
\text { COX-2 }\end{array}$ & COX-1/COX-2 & $\begin{array}{l}\text { IC } 50(\mu \mathrm{M}) \\
5 \mathrm{LOX}\end{array}$ \\
\hline Celecoxib & 5.47 & 0.86 & 7.91 & N.D \\
\hline $\begin{array}{l}\text { Meclofenamate } \\
\text { sodium }\end{array}$ & ND & ND & ND & 6.15 \\
\hline Compound $\mathbf{3}$ & 3.7 & 0.39 & 9.49 & 4.71 \\
\hline Compound 4 & 4.02 & 0.44 & 9.13 & 4.91 \\
\hline Compound $\mathbf{5}$ & 4.60 & 0.87 & 5.29 & 6.98 \\
\hline Compound 6 & 4.95 & 0.84 & 5.89 & 7.65 \\
\hline
\end{tabular}

orally given the newly synthesized compounds (3-6) in two dosages ( 5 and $10 \mathrm{mg} / \mathrm{kg}$ ). Results were expressed as rat paw edema percent. One hour later after administration of tested doses, carrageenan was injected sub planter in the left hind footpad of each rat as $0.05 \mathrm{ml}$ of $1 \%$ solution in sterile distilled water. Plethysmometer was used to measure paw edema volume from 0 to $4 \mathrm{~h}$ after carrageenan injection. Paw edema volume was compared with vehicle control group and reduction percent was calculated as the following

$$
\% \text { reduction in edema }=(1-\mathrm{Vt} \mathrm{Vc}) \times 100
$$

Where Vt and Vc are the edema volume in the group treated with drug and control, respectively [38]. Results were expressed as mean \pm standard deviation (SD). Differences between means were tested for significance using a one-way analysis of variance (ANOVA) followed by Duncan's test (Table 2).

\section{Antioxidant screening}

a. DPPH free radical scavenging assay was determined (4). Results were presented in Table 3 as IC ${ }_{50}(\mu \mathrm{g} /$ $\mathrm{ml}$ ). Ascorbic acid was used as reference standard antioxidant.

b. Lipid peroxidation assay ( $\mathbf{5}$ and $\mathbf{6}$ ) was calculated as $\mathrm{IC}_{50}$ and recorded in Table 3.

The newly synthesized compounds exhibited a remarkable in vivo and in vitro anti-inflammatory activity. These results are in agreement with those obtained by other researchers [39]. They reported that some novel pyrimidine-pyridine hybrids inhibited cyclooxygenase enzyme and had a significant anti-inflammatory activity comparable to celecoxib as a standard drug. In this concern, other authors [40] reported an investigation of the efficacy of pyridine and pyrimidine analog of acetaminophen as peroxyl radical trapping antioxidants and inhibitors of enzyme catalyzed lipid peroxidation by cyclooxygenase and lipoxygenase. Compounds $\mathbf{3}$ and $\mathbf{4}$ exhibited antioxidant activity screening higher scavenging activity towards the DPPH radicals than that of ascorbic acid. Similar results were reported for new pyridine and triazolopyridine derivatives [41-45].

Table 2 Inhibition percent of rat paw edema after administration of newly synthesized compounds

\begin{tabular}{|c|c|c|c|c|c|}
\hline Groups & $\mathrm{Oh}$ & $1 \mathrm{~h}$ & $2 \mathrm{~h}$ & $3 \mathrm{~h}$ & $4 h$ \\
\hline Diclofenac sodium & $0.49 \pm 0.032^{\mathrm{a}}$ & $30.22 \pm 1.27^{\mathrm{a}}$ & $33.85 \pm 1.19^{\mathrm{a}}$ & $36.21 \pm 0.93^{\mathrm{a}}$ & $41.10 \pm 3.98^{\mathrm{a}}$ \\
\hline $\begin{array}{l}\text { Compound } \mathbf{3} \\
5 \mathrm{mg} / \mathrm{kg} . b . w t\end{array}$ & $0.48 \pm 0.027^{\mathrm{ab}}$ & $21.72 \pm 0.79^{b}$ & $22.79 \pm 1.07^{d}$ & $24.79 \pm 0.49^{c}$ & $28.41 \pm 1.30^{c}$ \\
\hline $\begin{array}{l}\text { Compound } \mathbf{3} \\
10 \text { mg/kg.b.wt }\end{array}$ & $0.47 \pm 0.04^{\mathrm{ab}}$ & $31.98 \pm 9.35^{\mathrm{a}}$ & $29.93 \pm 1.43^{b}$ & $34.16 \pm 0.61^{b}$ & $41.15 \pm 0.750^{\mathrm{a}}$ \\
\hline $\begin{array}{l}\text { Compound } \mathbf{4} \\
5 \mathrm{mg} / \mathrm{kg} . b . w t\end{array}$ & $0.46 \pm 0.03^{b}$ & $18.91 \pm 1.19^{b c}$ & $20.07 \pm 1.43^{e}$ & $21.96 \pm 1.25^{d}$ & $27.38 \pm 1.68^{c}$ \\
\hline $\begin{array}{l}\text { Compound } \mathbf{4} \\
10 \mathrm{mg} / \mathrm{kg} . \mathrm{b} . \mathrm{wt}\end{array}$ & $0.49 \pm 0.01^{\mathrm{ab}}$ & $21.43 \pm 0.96^{b}$ & $26.27 \pm 49^{e}$ & $33.13 \pm 2.64^{b}$ & $37.15 \pm 0.69^{b}$ \\
\hline $\begin{array}{l}\text { Compound } \mathbf{5} \\
5 \mathrm{mg} / \mathrm{kg} . b . w t\end{array}$ & $0.49 \pm 0.01^{\mathrm{a}}$ & $11.62 \pm 1.24^{\text {de }}$ & $14.61 \pm 1.81^{9}$ & $18.41 \pm 1^{\mathrm{e}}$ & $19.48 \pm 0.89^{e}$ \\
\hline $\begin{array}{l}\text { Compound } \mathbf{5} \\
10 \mathrm{mg} / \mathrm{kg} . b . w t\end{array}$ & $0.48 \pm 0.01^{\mathrm{ab}}$ & $15.41 \pm 0.83^{\mathrm{cd}}$ & $18.83 \pm 0.75^{\mathrm{ef}}$ & $24.19 \pm 1.59^{c}$ & $28.59 \pm 2.26^{c}$ \\
\hline $\begin{array}{l}\text { Compound } 6 \\
5 \mathrm{mg} / \mathrm{kg} . b . w t\end{array}$ & $0.50 \pm 0.02^{\mathrm{a}}$ & $9.61 \pm 1.12^{\mathrm{e}}$ & $12.28 \pm 1.38^{h}$ & $17.19 \pm 1.26^{\mathrm{e}}$ & $18.51 \pm 2.26^{e}$ \\
\hline $\begin{array}{l}\text { Compound } \mathbf{6} \\
10 \mathrm{mg} / \mathrm{kg} . \mathrm{b} . \mathrm{wt}\end{array}$ & $0.49 \pm 0.01^{\mathrm{a}}$ & $13.75 \pm 1.15^{\text {de }}$ & $17.52 \pm 1.13^{f}$ & $20.43 \pm 0.65^{d}$ & $22.39 \pm 1.16^{d}$ \\
\hline
\end{tabular}

Values are expressed as mean \pm SD

Different superscript letters are significantly different at $P \leq 0.05$ 
Table 3 Showing antioxidant activities of the newly synthesized compounds

\begin{tabular}{lll}
\hline Groups & $\begin{array}{l}\mathbf{I C}_{\mathbf{5 0}}(\boldsymbol{\mu} \mathbf{g} / \mathbf{m l}) \text { for DPPH } \\
\text { Scavenging }\end{array}$ & $\begin{array}{l}\mathbf{I C}_{\mathbf{5 0}}(\boldsymbol{\mu g} / \mathbf{m l}) \text { for anti- } \\
\text { lipid peroxidation }\end{array}$ \\
\hline Compound $\mathbf{3}$ & $10.72 \pm 0.54$ & $16.81 \pm 2.71$ \\
Compound $\mathbf{4}$ & $12.64 \pm 0.41$ & $22.53 \pm 3.25$ \\
Compound $\mathbf{5}$ & $14.61 \pm 0.72$ & $23.62 \pm 2.31$ \\
Compound $\mathbf{6}$ & $15.26 \pm 0.44$ & $22.67 \pm 3.51$ \\
Ascorbic acid & $13.71 \pm 0.75$ & $25.72 \pm 1.23$ \\
\hline
\end{tabular}

\section{Experimental}

\section{Chemistry}

Melting points were measured using an Electrothermal IA 9100 equipment with open capillary tube and were kept uncorrected. All experiments were done using dry solvents. TLC was performed on Merck Silica Gel 60F254 with detection by way of UV Light. The formed compound has been purified using recrystallization. The IR spectra ( $\mathrm{KBr}$ disc) were recorded using Pye Unicam Sp-3-300 or a Shimadzu FTIR 8101 PC infrared spectrophotometer. The ${ }^{1} \mathrm{H}$ NMR and ${ }^{13} \mathrm{C}$ NMR spectra were measured by means of JEOL-JNM-LA $400 \mathrm{MHz}$ spectrometer using DMSO- $\mathrm{d}_{6}$ as a solvent. All chemical shifts had been expressed on the $\delta(\mathrm{ppm})$ scale using TMS as an internal well-known reference. The coupling constant $(J)$ values are given in $\mathrm{Hz}$. Analytical information was acquired from the Microanalysis center at Cairo University, Giza, Egypt.

\section{(E)-1-(pyridin-3-yl)-3-(thiophen-2-yl) prop-2-en-1-one (3)}

To a stirred mixture of thiophene-2-carbaldehyde $1(100 \mathrm{mmol})$ and 3-acetylpyridine $2(100 \mathrm{mmol})$ in $200 \mathrm{ml}$ ethanol at room temperature, $40 \% \mathrm{NaOH}$ aqueous solution was added portion-wise while stirring $2 \mathrm{~h}$. The pale yellow precipitate formed was filtered and washed using $4 \%$ aqueous $\mathrm{HCl}$, and crystallized from ethanol to give chalcone 3 in $82 \%$ yield, mp 256$258{ }^{\circ} \mathrm{C}$. IR (KBr) cm ${ }^{-1}$ : 3336, 3255, 1678, 1645; ${ }^{1} \mathrm{H}$ NMR (300 MHz, DMSO-d6): 6.93-6.96 (t, 1H, H5'-pyridine), 7.47-7.51 (dd, $1 H, H 3^{\prime}$-pyridine), 7.30-7.327(t, $1 \mathrm{H}$, H4' -pyridine), $7.53(\mathrm{~d}, 1 \mathrm{H}, J=12.9 \mathrm{~Hz},(\mathrm{C}=\mathrm{O})(\mathrm{CH}=\mathrm{C})$, $7.92(\mathrm{~d}, 1 \mathrm{H}, J=12.9 \mathrm{~Hz}(\mathrm{C}=\mathrm{O})(\mathrm{C}=\mathrm{CH}), \delta=7.28(\mathrm{~d}$, $1 \mathrm{H}, J=3.6 \mathrm{~Hz}$, thienyl- $\left.C_{3}{ }^{\prime} H\right), 7.94(\mathrm{dd}, 1 \mathrm{H}$, thienyl$\left.C_{4}{ }^{\prime} H\right), 8.11\left(\mathrm{~d}, 1 \mathrm{H}, J=5.2 \mathrm{~Hz}\right.$, thienyl- $\left.C_{5}{ }^{\prime} H\right) \cdot{ }^{13} \mathrm{C}$ NMR (DMSO- $\left.d_{6}, 150 \mathrm{MHz}\right): \delta=200.18(\mathrm{C} 1=\mathrm{O}) ; 153.3\left(\mathrm{C}^{\prime}{ }^{\prime}-\right.$ pyridine); 149.2 (C2'-pyridine); $147.4(\mathrm{C} 3) ; 135.2\left(\mathrm{C}^{\prime \prime}{ }^{\prime \prime}\right)$; 134.9 (C6'-pyridine); 133.2 (C'"-pyridine); $132.8\left(\mathrm{C}^{\prime \prime}{ }^{\prime \prime}\right)$; 127.1(C2); 126.8 (C5'-pyridine);125.4 (C3");123.6( $\left.\mathrm{C1}^{\prime \prime}\right)$. Anal. Calcd for $\mathrm{C}_{12} \mathrm{H}_{9} \mathrm{NOS}$ (215.27): C, 66.95; H, 4.21; N, 6.51; S, 14.90; Found C, 66.89; H, 4.19; N, 6.50; S, $14.79 \%$. 4-(pyridin-3-yl)-6-(thiophen-2-yl) pyrimidine-2(1H)-thione (4) Chalcone 3 (10 mmol) was added to sodium ethoxide solution [prepared from sodium metal $(0.23 \mathrm{~g}, 10 \mathrm{mmol})$ and $50 \mathrm{ml}$ of absolute ethanol] then thiourea $(10 \mathrm{mmol})$ was added. The reaction mixture was refluxed for $16 \mathrm{~h}$, left to cool and poured into crushed ice and neutralized with diluted hydrochloric acid, filtration, washed with ethanol and dried. Crystallization from EtOH afforded the pyrimidine derivatives 4 . Yellow powder, yield $74 \%$, mp 220-225 ${ }^{\circ} \mathrm{C}$; IR (KBr): $3433(\mathrm{NH}), 1270(\mathrm{C}=\mathrm{S}) \mathrm{cm}^{-1}$; ${ }^{1} \mathrm{H}$ NMR (300 MHz, DMSO-d $\left.{ }_{6}\right): \delta=6.93-6.96(\mathrm{t}, 1 \mathrm{H}$, H5'-pyridine), 7.47-7.51 (dd, $1 H, H 3^{\prime}$-pyridine), 7.30$7.327\left(\mathrm{t}, 1 \mathrm{H}, \mathrm{H} 4^{\prime}\right.$-pyridine $), 7.28(\mathrm{~d}, 1 \mathrm{H}, J=3.6 \mathrm{~Hz}$, thienyl$\left.C_{3}{ }^{\prime} H\right), 7.94\left(\mathrm{dd}, 1 \mathrm{H}\right.$, thienyl- $\left.C_{4}{ }^{\prime} H\right), 8.11(\mathrm{~d}, 1 \mathrm{H}, J=5.2 \mathrm{~Hz}$, thienyl- $\left.\mathrm{C}_{5}{ }^{\prime} H\right), 8.82\left(\mathrm{~s}, \mathrm{D}_{2} \mathrm{O}\right.$-exchangeable, $1 \mathrm{H}$, pyrimidin $\mathrm{NH}) .{ }^{13}$ CNMR (DMSO-d $6,100 \mathrm{MHz}$ ) $\delta: 110.2,123.9$, 127.1, 128.2, 130.5, 136.6, 137.2, 151.5, 152.0, 157.164.6, 180.4. Anal. Calcd for $\mathrm{C}_{13} \mathrm{H}_{9} \mathrm{~N}_{3} \mathrm{~S}_{2}$ (271.36): C, 57.54; H, 3.34; N, 15.48; S, 23.63; Found: C, 57.49; H, 3.32; N, 15.49; S, $23.59 \%$.

\section{1-(4-(pyridin-3-yl)-6-(thiophen-2-yl)pyrimidin-2-yl)hydrazine}

(5)

The reaction of thiopyrimidinone $4(10.0 \mathrm{mmol})$ with hydrazine hydrate $(10.0 \mathrm{mmol})$ catalyzed by acetic acid ( 5 drops) in refluxing ethanol for $6 \mathrm{~h}$. Evaporation of alcohol and recrystallization with ethanol gave compound 5 as pale brown crystals mp $180-182{ }^{\circ} \mathrm{C}$, yield $85 \%$. IR: $v \max / \mathrm{cm}^{-1}: 3212\left(\mathrm{NH}_{2}\right), 3184(\mathrm{NH}) .{ }^{1} \mathrm{H}$ NMR (DMSO$\left.\mathrm{d}_{6}\right): \delta=6.93-6.96\left(\mathrm{t}, 1 \mathrm{H}, \mathrm{H} 5^{\prime}\right.$-pyridine $), 7.47-7.51(\mathrm{dd}$, $1 H, \quad H 3^{\prime}$-pyridine), $7.30-7.327\left(\mathrm{t}, 1 \mathrm{H}, \quad \mathrm{H} 4^{\prime}\right.$-pyridine $)$, $\delta=7.28\left(\mathrm{~d}, 1 \mathrm{H}, J=3.6 \mathrm{~Hz}\right.$, thienyl- $\left.C_{3}{ }^{\prime} H\right), 7.53(\mathrm{dd}, 1 \mathrm{H}$, thienyl- $\left.C_{4}{ }^{\prime} H\right), 7.89\left(\mathrm{~d}, 1 \mathrm{H}, J=5.2 \mathrm{~Hz}\right.$, thienyl- $\left.C_{5}{ }^{\prime} H\right)$ 0.7.94 (s, $1 \mathrm{H}$, pyrimidin), 8.93-8.95 (brs, $3 \mathrm{H}, \mathrm{D}_{2} \mathrm{O}$ Exch., $\left.\mathrm{NH}_{2}, \mathrm{NH}\right) .{ }^{13} \mathrm{C}$ NMR (DMSO-d, $\left.100 \mathrm{MHz}\right) \delta: 98.4,123.9$, 127.1, 128.2, 130.5, 134.1, 137.2, 151.5, 152.0, 148.0, 149.1, 155.8, 157, 161.1. Anal.Calcd. For $\mathrm{C}_{13} \mathrm{H}_{11} \mathrm{~N}_{5} \mathrm{~S}$ (269.32): C, 57.97; H, 4.12; N, 26.00; S, 11.91; Found: C, 57.96; H, 4.09; N, $26.03 \%$.

\section{2-oxo-6-(pyridin-3-yl)-4-(thiophen-2-yl)-2H-pyran-3-carbon- itrile (6)}

To a stirred solution of chalcone $3(10 \mathrm{mmol})$ and ethyl cyanoacetate $(10 \mathrm{mmol})$ in $50 \mathrm{ml}$ absolute ethanol, a sodium ethoxide solution prepared from $0.23 \mathrm{~g}$ sodium metal $(10 \mathrm{mmol})$ and $10 \mathrm{ml}$ absolute ethanol was added refluxing the reaction mixture for $8 \mathrm{~h}$. The solid that formed after cooling was collected by filtration, washed with water, dried and finally crystallized from ethanol to afford compound 6 as pale yellow crystals in $72 \%$ yield, mp $210-212{ }^{\circ} \mathrm{C}$; IR $(\mathrm{KBr}): 2222(\mathrm{C} \equiv \mathrm{N}), 1688(\mathrm{C}=\mathrm{O})$ 
$\mathrm{cm}^{-1}$; ${ }^{1} \mathrm{H}$ NMR (300 MHz, DMSO-d6): $\delta=7.32(\mathrm{~s}, 1 \mathrm{H}$, C5), 6.93-6.96 (t, 1H, H5'-pyridine), 7.47-7.51 (dd, $1 H$, $H 3^{\prime}$-pyridine), 7.30-7.327(t, $1 \mathrm{H}, \mathrm{H} 4^{\prime}$-pyridine), 7.28(d, $1 \mathrm{H}, J=3.6 \mathrm{~Hz}$, thienyl- $\left.C_{3}{ }^{\prime} H\right), 7.94\left(\mathrm{dd}, 1 \mathrm{H}\right.$, thienyl- $\left.C_{4}{ }^{\prime} H\right)$, $8.11\left(\mathrm{~d}, 1 \mathrm{H}, J=5.2 \mathrm{~Hz}\right.$, thienyl- $\left.C_{5}{ }^{\prime} H\right) .{ }^{13} \mathrm{C}-\mathrm{NMR}$ (DMSO$\left.\mathrm{d}_{6}, 100 \mathrm{MHz}\right) \delta: 98.8,113.3,115.9,123.8,127.1,128.2$, 130.5, 137.7, 136.6, 149.6, 157.4, 173.4. Anal. Calcd for $\mathrm{C}_{15} \mathrm{H}_{8} \mathrm{~N}_{2} \mathrm{O}_{2} \mathrm{~S}$ (280.3): C, 64.27; H, 2.88; N, 9.99; S, 11.44; Found: C, 64.25; H, 2.84; N, 9.96; S, 11.42\%.

\section{1-amino-6-oxo-4-(thiophen-2-yl)-1,6-dihydro-[2,3'-bipyridin e]-5-cabonitrile (7)}

To a solution of the pyranone $6(2 \mathrm{mmol})$ in $30 \mathrm{ml}$ of ethanol, hydrazine hydrate $(2 \mathrm{mmol})$ was added. The mixture was refluxed for $6 \mathrm{~h}$. Left to cool, the formed solid product was filtered off, dried, and then crystallized from ethanol to give compounds 7 . Yellow powder, yield 70\%, mp 250-252 ${ }^{\circ} \mathrm{C}$; IR ( $\left.\mathrm{KBr}\right): 3320,3190\left(\mathrm{NH}_{2}, \mathrm{NH}\right)$, $2219(\mathrm{C} \equiv \mathrm{N}), 1670(\mathrm{C}=\mathrm{O}) \mathrm{cm}^{-1} ;{ }^{1} \mathrm{H}$ NMR $(300 \mathrm{MHz}$, DMSO- $\left.\mathrm{d}_{6}\right): \delta=2.51\left(\mathrm{~s}, \mathrm{D}_{2} \mathrm{O}\right.$-exchangeable, $2 \mathrm{H}, \mathrm{NH}_{2}$ ), 6.93-6.96 (t, 1H, H5'-pyridine), 7.47-7.51 (dd, $1 H, H 3^{\prime}$ pyridine), 7.30-7.327(t, $1 \mathrm{H}, \mathrm{H} 4^{\prime}$-pyridine $), 7.28(\mathrm{~d}, 1 \mathrm{H}$, $J=3.6 \mathrm{~Hz}$, thienyl- $\left.C_{3}{ }^{\prime} H\right), 7.94$ (dd, $1 \mathrm{H}$, thienyl- $\left.C_{4}{ }^{\prime} H\right)$, $8.11\left(\mathrm{~d}, 1 \mathrm{H}, J=5.2 \mathrm{~Hz}\right.$, thienyl- $\left.C_{5}{ }^{\prime} H\right) .{ }^{13} \mathrm{C}-\mathrm{NMR}$ (DMSO$\left.\mathrm{d}_{6}, 100 \mathrm{MHz}\right) \delta: 110.8,115.9,121.3,123.8,127.1,128.2$, 130.5, 131.6, 136.8, 149.6, 150.0, 160.5, 169.4. Anal. Calcd for $\mathrm{C}_{15} \mathrm{H}_{10} \mathrm{~N}_{4} \mathrm{OS}$ (294.33): C, 61.21; $\mathrm{H}, 3.42 ; \mathrm{N}, 19.04 ; \mathrm{S}$, 10.89; Found: C, 61.20; H, 3.40; N, 19.09; S, 10.90\%.

\section{3,4-dimethyl-1-(4-(pyridin-3-yl)-6-(thiophen-2-yl)pyrimi- \\ din-2-yl)pyrano[2,3-c]pyrazol-6(1H)-one (8)}

Compound $5(10 \mathrm{mmol})$ and ethyl acetoacetate in excess $(30 \mathrm{ml})$ was heated at reflux temperature for $6 \mathrm{~h}$. The mixture was poured into ice cold water and the obtained product washed with water, dried and recrystallized from ethanol to give pale brown crystals of the pyrazolone compound $8 \mathrm{mp} 190-192{ }^{\circ} \mathrm{C}$, yield $80 \%$ IR: vmax $/ \mathrm{cm}^{-1}$ $3367(\mathrm{NH}), 1715(\mathrm{C}=\mathrm{O}) .{ }^{1} \mathrm{H}$ NMR $\left(\mathrm{DMSO}_{6} \mathrm{~d}_{6}\right): \delta 2.25$ (s, $\left.3 \mathrm{H}, \mathrm{CH}_{3}\right), 2.32\left(\mathrm{~s}, 3 \mathrm{H}, \mathrm{CH}_{3}\right), 5.60(\mathrm{~s}, 1 \mathrm{H}$, pyranone), 6.93-6.96 (t, 1H, H5'-pyridine), 7.47-7.51 (dd, $1 H, H 3^{\prime}$ pyridine), 7.30-7.327(t, $1 \mathrm{H}, \mathrm{H} 4^{\prime}$-pyridine), 7.28(d, $1 \mathrm{H}$, $J=3.6 \mathrm{~Hz}$, thienyl- $\left.C_{3}{ }^{\prime} H\right), 7.94\left(\mathrm{dd}, 1 \mathrm{H}\right.$, thienyl- $\left.C_{4}{ }^{\prime} H\right)$, $8.11\left(\mathrm{~d}, 1 \mathrm{H}, J=5.2 \mathrm{~Hz}\right.$, thienyl- $\left.C_{5}{ }^{\prime} H\right) .{ }^{13} \mathrm{C}-\mathrm{NMR}$ (DMSO$\left.\mathrm{d}_{6}, 100 \mathrm{MHz}\right) \delta: 11.9,21.2,101.5,105,118.5,124.0,125.5$, 127.9, 33.1, 134.1, 141.7, 148.0, 149.1, 152.8, 155.6, 159.0, 160.9, 161.0. Anal. Calcd. For $\mathrm{C}_{21} \mathrm{H}_{15} \mathrm{~N}_{5} \mathrm{O}_{2} \mathrm{~S}$ (401.44): C, 62.83; H, 3.77; N, 17.45; S, 7.99; Found: C, 62.79; H, 3.79; N, 17.47; S, 7.95\%. 3-methyl-1-(4-(pyridin-3-yl)-6-(thiophen-2-yl) pyrimidin-2-yl)-1H-pyrazol-5(4H)-one (9)

Compound 5 (10 $\mathrm{mmol})$ and ethyl acetoacetate $(10 \mathrm{mmol})$ in acetic acid $(30 \mathrm{ml})$ was heated at reflux temperature for $6 \mathrm{~h}$. The mixture was poured into ice cold water and the obtained product washed with ice cold water, dried and recrystallized from ethanol to afford pale brown crystals of $9 \mathrm{mp} 225-227{ }^{\circ} \mathrm{C}$, yield 76\% IR: vmax/ $\mathrm{cm}^{-1}$ : $3216(\mathrm{NH}), 1718(\mathrm{C}=\mathrm{O}) .{ }^{1} \mathrm{H}$ NMR (DMSO-d6): $\delta$ $1.20\left(\mathrm{~s}, 3 \mathrm{H}, \mathrm{CH}_{3}\right), 2.25\left(\mathrm{~s}, 2 \mathrm{H}, \mathrm{CH}_{2}\right.$, pyrazol), 6.93-6.96 (t, $1 \mathrm{H}, \mathrm{H} 5^{\prime}$-pyridine), 7.47-7.51 (dd, $1 \mathrm{H}, \mathrm{H3}^{\prime}$-pyridine), 7.30-7.327(t, $1 \mathrm{H}, \mathrm{H} 4^{\prime}$-pyridine), 7.28(d, $1 \mathrm{H}, J=3.6 \mathrm{~Hz}$, thienyl- $\left.C_{3}{ }^{\prime} H\right)$, $7.94\left(\mathrm{dd}, 1 \mathrm{H}\right.$, thienyl- $\left.C_{4}{ }^{\prime} H\right), 8.11(\mathrm{~d}, 1 \mathrm{H}$, $J=5.2 \mathrm{~Hz}$, thienyl- $\left.C_{5}{ }^{\prime} H\right), 8.90$ (s, $1 \mathrm{H}$, pyrimidin) ${ }^{13} \mathrm{C}-$

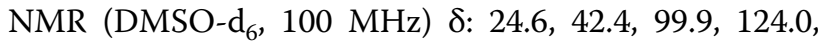
$125.5,127.6,133.1,134.1,140,148.0,149.1,156.1$, 160.2 , 163.1, 159.5, 172.8. Anal. Calcd. For $\mathrm{C}_{17} \mathrm{H}_{13} \mathrm{~N}_{5} \mathrm{OS}$ (335.38): C, 60.88; H, 3.91; N, 20.88; S, 9.56; Found: C, $60.90 ; \mathrm{H}, 3.90 ; \mathrm{N}, 20.86 ; \mathrm{S}, 9.55 \%$.

\section{2-(3,5-dimethyl-1H-pyrazol-1-yl)-4-(pyridin-3-yl)-6-(thiophen -2-yl)pyrimidine (10)}

A solution of compound 5 (10 mmol) in absolute ethanol and acetylacetone $(10 \mathrm{mmol})$ was heated at reflux temperature for $5 \mathrm{~h}$. the obtained product was recrystallized from ethanol to afford pale brown crystals of pyrazolo pyrimidine derivative 10. mp $190-188{ }^{\circ} \mathrm{C}$, yield $70 \%$ IR: $\operatorname{vmax} / \mathrm{cm}^{-1} 3170(\mathrm{NH}), 1600(\mathrm{C}=\mathrm{N}), 1574(\mathrm{C}=\mathrm{N}) .{ }^{1} \mathrm{H}$ NMR (DMSO-d6): $\delta 2.3\left(\mathrm{~s}, 3 \mathrm{H}, \mathrm{CH}_{3}\right), 2.58\left(\mathrm{~s}, 3 \mathrm{H}, \mathrm{CH}_{3}\right)$, 6.99 (s, $1 H$, pyrazole), 6.93-6.96 (t, $1 \mathrm{H}, \mathrm{H} 5^{\prime}$-pyridine), 7.47-7.51 (dd, $1 H, H 3^{\prime}$-pyridine), 7.30-7.327(t, 1H, H4'pyridine), $7.28\left(\mathrm{~d}, 1 \mathrm{H}, J=3.6 \mathrm{~Hz}\right.$, thienyl- $\left.C_{3}{ }^{\prime} H\right), 7.94(\mathrm{dd}$, $1 \mathrm{H}$, thienyl- $\left.C_{4}{ }^{\prime} H\right), 8.11\left(\mathrm{~d}, 1 \mathrm{H}, J=5.2 \mathrm{~Hz}\right.$, thienyl- $\left.C_{5}{ }^{\prime} H\right)$, 8.94 (s, $1 \mathrm{H}$, pyrimidine). ${ }^{13} \mathrm{C}-\mathrm{NMR}$ (DMSO-d6, $100 \mathrm{MHz}$ ) $\delta: 11.1,18,105,125.5,127.6,133.1,140,144.3,148.0$, 149.1, 155.6, 159.0, 161.0. Anal. Calcd. For $\mathrm{C}_{18} \mathrm{H}_{15} \mathrm{~N}_{5} \mathrm{~S}$ (333.41): C, 64.84; H, 4.53; N, 21.01; S, 9.62; Found: C, 64.80; H, 4.52; N, 21.02, S, 9.60\%.

\section{Conclusions}

We have reported the synthesis of (E)-1-(pyridin-3-yl)-3(thiophen-2-yl) prop-2-en-1-one 3 and using to designing a polycyclic heterocyclic compounds containing five and/ or six rings fused. Moreover, we concluded that compounds $\mathbf{3}$ and $\mathbf{4}$ showed a significant antioxidant activity regarding cyclooxygenase inhibitory activity, compound 3 presented the highest inhibitory activity in comparison to the standard reference drug $\left[\mathrm{IC}_{50}\right.$ as 3.7 and $0.39 \mu \mathrm{M}$ for COX-1 and COX-2, respectively compared to 5.47 and 0.86 for the standard celecoxib]. Compound $\mathbf{4}$ also 
showed a potent inhibitory activity for COX-2 with $\mathrm{IC}_{50}$ 0.44. Compounds 5 and $\mathbf{6}$ showed inhibitory activity against COX-1 and COX-2 nearly like that of the standard drug. Compound 3 showed the highest inhibitory potential for 5-lipoxygenase with $\mathrm{IC}_{50}(4.71 \mu \mathrm{M})$ compared to $(6.15 \mu \mathrm{M})$ of the standard anti-inflammatory drug meclofenamate sodium.

\section{Abbreviations}

EtOH: ethanol; NMR: nuclear magnetic resonance; IR: infrared radiation; DMSO: dimethyl sulfoxide; COX-1: cyclooxygenase-l; COX-2: cyclooxygenase-II; 5-LOX: 5-lipoxygenase; DPPH: 2, 2-diphenyl-1-picrylhydrazil.

\section{Authors' contributions}

WSS carried the literature and designed synthetic schemes (synthesis and purification). SMM contributed to study of anti-inflammatory activities both in vitro and in vivo and Antioxidant vitalities against a, a-diphenyl$\beta$-picrylhydrazyl (DPPH) scavenging activity and lipid peroxidation. MHA records the ${ }^{13} \mathrm{CNMR}$ of all compounds. All authors read and approved the final manuscript.

\section{Author details \\ ${ }^{1}$ Department of Chemistry, Faculty of Science, Zagazig University, Zaga- zig 44519, Egypt. ${ }^{2}$ Department of Pharmaceutical Chemistry, Deanship of Scientific Research, Taif University, Taif 21974-888, Kingdom of Saudi Arabia. ${ }^{3}$ Departments of Pharmacology, Faculty of Veterinary Medicine, Cairo Univer- sity, Cairo 12211, Egypt.}

\section{Acknowledgements}

The authors are very thankful to all the associated personnel in any reference that contributed in/for the purpose of this research.

\section{Competing interests}

The authors declare that they have no competing interests

\section{Consent for publication}

All authors consent to publication.

\section{Funding}

This research is not funded though any source.

\section{Ethics approval and consent to participate}

Not applicable.

\section{Publisher's Note}

Springer Nature remains neutral with regard to jurisdictional claims in published maps and institutional affiliations.

\section{Received: 24 April 2018 Accepted: 2 June 2018}

Published online: 08 June 2018

\section{References}

1. Firoozpour L, Edraki N, Nakhjiri M, Emami S, Safavi M, Ardestani SK, Khoshneviszadeh M, Shfiee A, Foroumadi A (2012) Cytotoxic activity evaluation and QSAR study of chromene-based chalcone. Arch Pharm Res 35:2117-2125

2. Baviskar BA, Baviskar B, Shiradkar MR, Deokate UA, Khadabadi SS (2009) Synthesis and antimicrobial activity of some novel. Benzimidazolyl chalcones. Eur J Chem 6:196-200

3. Munawar MA, Azad M, Siddiqui HL (2008) Synthesis and antimicrobial studies of some quinolinylpyrimidine derivatives. J Chin Soc 55:394-400

4. Azad M, Munawar MA, Siddiqui HL (2007) Antimicrobial activity and synthesis of quinoline-base chalcones. J Appl Sci 7:2485-2489
5. Kalirajan R, Sivakumar SU, Jubie S, Gowramma B, Suresh B (2009) Synthesis and biological evaluation of some heterocyclic derivatives of chalcones. Int J ChemTech Res 1:27-34

6. Talia JM, Debattista NB, Pappano NB (2011) New antimicrobial combinations: substituted chalcones-oxacillin against methicillin resistant Staphylococcus aureus. Braz J Microbiol 42:470-475

7. Eumkeb G, Siriwong S, Phitaktim S, Rojtinnakorn N, Sakdarat S (2012) Synergistic activity and mode of action of flavonoids isolated from smaller galangal and amoxicillin combinations against amoxicillin-resistant Escherichia coli. J Appl Microbiol 112:55-64

8. Do TH, Nguyen DM, Truong VD, Do THT, Le MT, Pham TQ, Thai KM, Tran TD (2016) Synthesis and selective cytotoxic activities on rhabdomyosarcoma and noncancerous cells of some heterocyclic chalcones. Molecules 21(3):329. https://doi.org/10.3390/molecules21030329

9. Sato Y, Shibata H, Arakaki N, Higuti T (2004) 6,7-dihydroxyflavone dramatically intensifies the susceptibility of methicillin-resistant or -sensitive Staphylococcus aureus to beta-lactams. Antimicrob Agents Chemother 48:1357-1360

10. Babasaheb PB, Sachin AP, Rajesh NG (2010) Synthesis and biological evaluation of nitrogencontaining chalcones as possible anti-inflammatory and antioxidant agents. Bioorg Med Chem Lett 20:730-733

11. Vogel S, Barbic M, Jürgenliemk G, Heilmann J (2010) Synthesis, cytotoxicity, anti-oxidative and anti-inflammatory activity of chalcones and influence of A-ring modifications on the pharmacological effect. Eur J Med Chem 45:2206-2213

12. Tran T-D, Park H, Kim HP, Ecker GF, Thai K-M (2009) Inhibitory activity of prostaglandin E2 production by the synthetic 21-hydroxychalcone analogues: synthesis and SAR study. Bioorg Med Chem Lett 19:1650-1653

13. Kim BT, Kwang-Joong O, Chun JC, Hwang KJ (2008) Synthesis of dihydroxylated chalcone derivatives with diverse substitution patterns and their radical scavenging ability toward DPPH free radicals. Bull Korean Chem Soc 29:1125-1130

14. Doan TN, Tran T-D (2011) Synthesis, antioxidant and antimicrobial activities of a novel series of chalcones, pyrazolic chalcones, and allylic chalcones. Pharmacol Pharm 2:282-288

15. Sivakumar PM, Prabhakar PK, Doble M (2011) Synthesis, antioxidant evaluation, and quantitative structure-activity relationship studies of chalcones. Med Chem Res 20:482-492

16. Vogel S, Ohmayer S, Brunner G, Heilmann J (2008) Natural and nonnatural prenylated chalcones: synthesis, cytotoxicity and anti-oxidative activity. Bioorg Med Chem 16:4286-4293

17. Echeverria C, Santibañez JF, Donoso-Tauda O, Escobar CA, Ramirez-Tagle $R$ (2009) Structural antitumoral activity relationships of synthetic chalcones. Int J Mol Sci 10:221-231

18. Modzelewska A, Pettit C, Achanta G, Davidson NE, Huang P, Khan SR (2006) Anticancer activities of novel chalcone and bis-chalcone derivatives. Bioorg Med Chem 14:3491-3495

19. Vogel S, Heilmann J (2008) Synthesis, cytotoxicity, and antioxidative activity of minor prenylated chalcones from Humulus lupulus. J Nat Prod 71:1237-1241

20. Kamal A, Kashi Reddy M, Viswanath A (2013) The design and development of imidazothiazole-chalcone derivatives as potential anticancer drugs. Expert Opin Drug Discov 8:289-304

21. Do TH, Nguyen DM, Truong VD, Do THT, Le MT, Pham TQ, Thai KM, Tran TD (2016) Synthesis and selective cytotoxic activities on rhabdomyosarcoma and noncancerous cells of some heterocyclic chalcones. Molecule 21:329. https://doi.org/10.3390/molecules21030329

22. Neves MP, Lima RT, Choosang K, Pakkong P, de São José Nascimento M, Vasconcelos MH, Pinto M, Silva AM, Cidade H (2012) Synthesis of a natural chalcone and its prenyl analogs-evaluation of tumor cell growth-inhibitory activities, and effects on cell cycle and apoptosis. Chem Biodivers 9:1133-1143

23. Forejtníková $H$, Lunerová $K$, Kubínová R, Jankovská D, Marek R, Suchý V, Vondrácek J (2005) Chemoprotective and toxic potentials of synthetic and natural chalcones and dihydrochalcones in vitro. Toxicology 208:81-93

24. Orlikova B, Tasdemir D, Golais F, Dicato M, Diederich M (2011) Dietary chalcones with chemopreventive and chemotherapeutic potential. Genes Nutr 6:125-147

25. Ekhlass N, El-Badry YA, Eltoukhy AMM, Ayyad RR (2016) Synthesis and antiproliferative activity of 1-(4-(1H-Indol-3-YI)-6-(4-Methoxyphenyl) 
Pyrimidin-2-yl)hydrazine and its pyrazolo pyrimidine derivatives. Medicinal chemistry. Med chem 6:4. https://doi.org/10.4172/2161-0444.1000350

26. Murakami N, Takase H, Saito T, Iwata K, Miura H, Naruse T (1998) Effects of a novel non-steroidal anti-inflammatordrug (M-5011) on bone metabolism in rats with collageninduced arthritis. Eur J Pharmacol 352(1):81-90

27. Kulandasamy R, Adhikari AV, Stables JP (2009) A new class of anticonvulsants possessing $6 \mathrm{~Hz}$ activity: 3,4-dialkyloxy thiophene bishydrazones. Eur J Med Chem 44(11):4376-4384

28. Lu X, Wan B, Franzblau SG, You Q (2011) Design, synthesis and antitubercular evaluation of new 2-acylated and 2-alkylated amino-5-(4(benzyloxy) phenyl)thiophene-3-carboxylic acid derivatives. Part 1. Eur J Med Chem 46(9):3551-3563

29. Kaushik NK, Kim HS, Chae YJ et al (2012) Synthesis and anticancer activity of di(3-thienyl)methanol and di(3-thienyl)methane. Molecules 17(10):11456-11468

30. Shehab WS, Saad HA, Mouneir SM (2017) Synthesis and antitumor/ antiviral evaluation of 6-thienyl-5-cyano-2-thiouracil derivatives and their thiogalactosides analogs. Curr Org Synth 14:291-298

31. Shehab WS, Mouneir SM (2015) Design, synthesis, antimicrobial activity and anticancer screening of some new1,3-thiazolidin-4-ones derivatives. Eur J Chem 6:157

32. Shehab WS (2009) Synthesis of tetrahydropyrimidine derivatives and its glycosides. Curr Org Chem 13:14

33. Corral C, Lissavetzky J, Manzanares I et al (1999) Synthesis and preliminary pharmacological evaluation of thiophene analogues of viloxazine as potentialantidepressant drugs. Bioorg Med Chem 7(1):349-1359

34. Abdel-Wahab BF, Abdel-Aziz HA, Ahmed EM (2009) Synthesis and antimicrobial evaluation of 1-(benzofuran-2-yl)-4-nitro-3-arylbutan-1-ones and 3-(benzofuran-2-yl)-4, 5-dihydro-5-aryl-1-[4-(aryl)-1, 3-thiazol-2-yl]1 H-pyrazoles. Eur J Med Chem 44:2632-2635

35. Kulmacz RJ, Lands WEM (1983) Requirements for hydroperoxide by the cyclooxygenase and peroxidase activities of prostaglandin $\mathrm{H}$ synthase. Prostaglandins 25:531-540
36. Jacob J, Prakash Kumar B (2015) Dual COX/LOX inhibition: screening and evaluation of effect of medicinal plants of Kerala as Antiinflammatory agents Pharmacogn. Phytochemistry 3:62-66

37. Kuroda T, Suzuki F, Tamura T, Ohmori K, Hosoe H (1992) A novel synthesis and potent antiinflammatory activity of 4-hydroxy-2(1H)-oxo-1-phenyl1,8-naphthyridine-3-carboxamides. J Med Chem 36:1130-1136

38. Simone RD, Chini MG, Bruno I, Riccio R (2011) Benzimidazole-1,2,3-triazole hybrid molecules: synthesis and evaluation for antibacterial/antifungal activity. J Med Chem 54:1565-1575

39. Khalil NA, Ahmed EM, Mohamed KO, Nissan YM (2014) Synthesis and biological evaluation of new pyrazolone-pyridazine conjugates as antiinflammatory and analgesic agents. Bioorg Med Chem 22:2080-2089

40. Lokwani D, Azad R, Sarkate A, Reddanna P, Shinde D (2015) Structure based library design (SBLD) for new 1, 4-dihydropyrimidine scaffold as simultaneous COX-1/COX-2 and 5-LOX inhibitors. Bioorg Med Chem 23:4533-4543

41. Abdelgawad MA, Bakr RB, Azouz AA (2018) Novel pyrimidine-pyridine hybrids: synthesis, cyclooxygenase inhibition, anti-inflammatory activity and ulcerogenic liability. Bioorg Chem 77:339-348

42. Nam TG, Nara SJ, Zagol-Ikapitte I, Cooper T, Valgimigli L, Oates JA, Porter NA, Boutaud O, Pratt DA (2009) Pyridine and pyrimidine analogs of acetaminophen as inhibitors of lipid peroxidation and cyclooxygenase and lipoxygenase catalysis. Org Biomol Chem 7(24):5103-5112

43. Kotb ER, Soliman HA, Morsy EMH, Abdelwahed NAM (2017) New pyridine and triazolopyridine derivatives: synthesis, antimicrobialand antioxidant evaluation. Acta Pol Pharm 74(3):861-872

44. Mojarrab M, Soltani R, Aliabadi A (2013) Pyridine based chalcones: synthesis and evaluation of antioxidant activity of 1-phenyl-3-(pyridin-2-yl) prop-2-en-1-one derivatives. Jundishapur J Nat Pharm Prod 8(3):125-130

45. Rani J, Saini M, Kumar S, Verma PK (2017) Design, synthesis and biological potentials of novel tetrahydroimidazo[1,2-a]pyrimidinederivatives. Chem Cent J 11:16

\section{Submit your manuscript to a SpringerOpen ${ }^{\circ}$ journal and benefit from:}

- Convenient online submission

- Rigorous peer review

- Open access: articles freely available online

- High visibility within the field

Retaining the copyright to your article

Submit your next manuscript at $\boldsymbol{\nabla}$ springeropen.com 